\title{
A deep learning quantified stroma-immune score to predict survival of patients with stage II-III colorectal cancer
}

Zeyan $\mathrm{Xu}^{1,2+}$, Yong $\mathrm{Li}^{3 \dagger}$, Yingyi Wang ${ }^{4 \dagger}$, Shenyan Zhang ${ }^{5 \dagger}$, Yanqi Huang ${ }^{1}$, Su Yao ${ }^{6}$, Chu Han ${ }^{1}$, Xipeng Pan ${ }^{1}$, Zhenwei Shi ${ }^{1}$, Yun Mao ${ }^{7}$, Yao Xu ${ }^{8}$, Xiaomei Huang ${ }^{1,9}$, Huan Lin ${ }^{1,2}$, Xin Chen ${ }^{10}$, Changhong Liang ${ }^{1}$, Zhenhui Li ${ }^{1,5^{*}}$, Ke Zhao ${ }^{1 *}$, Qingling Zhang ${ }^{6 *}$ and Zaiyi Liu ${ }^{1,2^{*}}$ (D)

\begin{abstract}
Background: Profound heterogeneity in prognosis has been observed in colorectal cancer (CRC) patients with intermediate levels of disease (stage II-III), advocating the identification of valuable biomarkers that could improve the prognostic stratification. This study aims to develop a deep learning-based pipeline for fully automatic quantification of immune infiltration within the stroma region on immunohistochemical (IHC) whole-slide images (WSIs) and further analyze its prognostic value in CRC.

Methods: Patients from two independent cohorts were divided into three groups: the development group ( $N=200)$, the internal $(N=134)$, and the external validation group $(N=90)$. We trained a convolutional neural network for tissue classification of CD3 and CD8 stained WSIs. A scoring system, named stroma-immune score, was established by quantifying the density of $\mathrm{CD}^{+}$and $\mathrm{CD} 8^{+}$T-cells infiltration in the stroma region.

Results: Patients with higher stroma-immune scores had much longer survival. In the development group, 5-year survival rates of the low and high scores were $55.7 \%$ and $80.8 \%$ (hazard ratio [HR] for high vs. low $0.39,95 \%$ confidence interval $[\mathrm{Cl}] 0.24-0.63, \mathrm{P}<0.001)$. These results were confirmed in the internal and external validation groups with 5 -year survival rates of low and high scores were $57.1 \%$ and $78.8 \%, 63.9 \%$ and $88.9 \%$, respectively (internal: HR for high vs. low $0.49,95 \% \mathrm{Cl} 0.28-0.88, \mathrm{P}=0.017$; external: $\mathrm{HR}$ for high vs. low $0.35,95 \% \mathrm{Cl} 0.15-0.83, \mathrm{P}=0.018)$. The combination of stroma-immune score and tumor-node-metastasis (TNM) stage showed better discrimination ability for survival prediction than using the TNM stage alone.
\end{abstract}

Conclusions: We proposed a stroma-immune score via a deep learning-based pipeline to quantify $\mathrm{CD}^{+}$and $\mathrm{CD} 8^{+}$ T-cells densities within the stroma region on WSIs of CRC and further predict survival.

Keywords: Deep learning, Whole-slide images, Tumor-infiltrating lymphocytes, Colorectal cancer

*Correspondence: lizhenhui621@qq.com; ksw2024@163.com; zqllc8@126. com; liuzaiyi@gdph.org.cn

†Zeyan Xu, Yong Li, Yingyi Wang and Shenyan Zhang contributed equally to this work

${ }^{1}$ Department of Radiology, Guangdong Provincial People's Hospital, Guangdong Academy of Medical Sciences, 106 Zhongshan Er Road, Guangzhou 510080, China

${ }^{6}$ Department of Pathology, Guangdong Provincial People's Hospital, Guangdong Academy of Medical Sciences, Guangzhou 510080, China Full list of author information is available at the end of the article

\section{Introduction}

Colorectal cancer (CRC) is one of the leading causes of cancer-associated death worldwide [1]. Currently, therapeutic decisions and prognostic evaluations of CRC are mainly performed by the tumor-node-metastasis (TNM) staging system [2]. However, the TNM staging system fails to provide complete prognostic information as diverse prognoses are observed among patients original author(s) and the source, provide a link to the Creative Commons licence, and indicate if changes were made. The images or other third party material in this article are included in the article's Creative Commons licence, unless indicated otherwise in a credit line to the material. If material is not included in the article's Creative Commons licence and your intended use is not permitted by statutory regulation or exceeds the permitted use, you will need to obtain permission directly from the copyright holder. To view a copy of this licence, visit http://creativecommons.org/licenses/by/4.0/. The Creative Commons Public Domain Dedication waiver (http://creativeco mmons.org/publicdomain/zero/1.0/) applies to the data made available in this article, unless otherwise stated in a credit line to the data. 
with the same stage due to the differences in clinical and molecular phenotypes, patterns of genetic damage, and host immune responses [3, 4]. In particular, although the treatment strategies such as surgery, chemotherapy, radiotherapy, and immunotherapy have improved obviously, profound heterogeneity in prognosis has been observed in CRC patients with intermediate levels of disease (stage II-III) [4]. Hence, biomarkers that could improve the prognostic stratification for patients with stage II-III CRC are urgently needed.

The increased knowledge of the immune system's central role in tumor progression advocates identifying prognostic biomarkers to describe immune infiltration. In particular, the Immunoscore, which is obtained from the densities of $\mathrm{CD}^{+}$and $\mathrm{CD}^{+}$T-cells in the tumor center and invasive margin, has been reported to hold superior and independent prognostic value over the traditional TNM system in patients with stage II-III CRC $[5,6]$. Recently, a more detailed immune infiltration analysis approach has been proposed to describe the quantitative landscape of tumor-immune microenvironment (TIME) in CRC by quantifying tumorinfiltrating lymphocytes (TILs) in the stromal and intraepithelial regions, respectively, with inspiring results [7]. This advocate further interest in the tumor immune cell infiltration in one of the major constituents of the TIME, the stroma. Additionally, as shown by the study of Kather et al. [8], the information from non-tumor components (such as stroma) could provide more prognostic value than tumor epithelium. The tumor stroma characteristics, such as the tumorstroma ratio (TSR), have been well supported by emerging studies as an independent prognostic tool in CRC $[9,10]$.

With the recent advance of artificial intelligence technologies and digital whole-slide images (WSIs), it is possible to identify novel biomarkers from automatically segmented histological components [11]. Previous studies conducted automatic quantification of TILs based on multiplex immunofluorescence WSIs, suggesting that TILs combined with other risk factors can improve the accuracy of prognosis prediction in CRC patients [12, 13]. Therefore, inspired by previous observations [5, 7 , 10], we postulate that describing the immune infiltration lymphocytes $\left(\mathrm{CD}^{+}\right.$and $\mathrm{CD} 8^{+} \mathrm{T}$-cells) in the stroma region using a deep learning approach could further refine the prognostic stratification of patients with stage II-III CRC.

Therefore, the goal of this study is to propose a deep learning-based pipeline for fully automated quantification of immune infiltration within the stroma region on the immunohistochemical (IHC) WSIs and further analyze its prognostic value in patients with CRC.

\section{Methods}

\section{Patients and follow up}

This retrospective study was approved by the Research Ethics Committee of Guangdong Provincial People's Hospital (Cohort 1) and the Sixth Affiliated Hospital of Sun Yat-sen University (Cohort 2), and the informed consent was waived. The institutional medical record database was analyzed to identify patients with histologically confirmed stage II-III CRC patients who underwent surgical resection with curative intent from Mar 2009 to Dec 2014 at Cohort 1 and Jan 2013 to Dec 2014 at Cohort 2. Patients with follow-up information and IHC (CD3 and CD8) WSIs available were included in the study. Patients who received neoadjuvant therapy or died within 30 days after surgery were excluded. Moreover, patients with incomplete clinical information and poor image quality were also excluded. After enrollment, patients from Cohort 1 were randomly divided into two groups: $60 \%$ of patients formed the development group, whereas $40 \%$ formed the internal validation group. Patients from Cohort 2 formed the external validation group.

Clinicopathological factors were collected from medical records, including age, sex, tumor site (colon/rectum), T-category, N-category, TNM stage, microsatellite instability (MSI) status, and treatment modalities. MSI status was determined by IHC with the expression of mismatch repair proteins (MLH1, MSH2, MSH6, and PMS2) and classified as MSI and microsatellite stable (MSS). The outcome of interest was overall survival (OS). The follow-up methods included clinical consultations, medical records reviews, and telephone interviews.

\section{Datasets for tissue classification}

The CD3 and CD8 IHC stained tissue sections were imaged using digital Whole Slide Scanning (AperioAT2, Leica, USA) at $40 \times$ magnification. CRC tissues were grouped into nine types: tumor epithelium, tumor stroma, adipose, background, debris, lymphocytes, mucus, smooth muscle, and normal mucosa. For decomposing different tissue types on IHC WSIs, we used two tiles datasets to train a tissue classification model and one tile dataset to test the model. An open available hematoxylin and eosin (HE) tiles dataset consisting of $283 \mathrm{k}$ tissue tiles was used as the HE tiles dataset $[8,10]$. An IHC tiles training dataset consisting of $154.4 \mathrm{k}$ tissue tiles was established from 242 CD3 and CD8 slides of 121 patients in the development group. An IHC tiles test dataset consisting of $22.5 \mathrm{k}$ tissue tiles was also established from 114 slides of 57 patients in the internal validation group. Details of the IHC staining and datasets generation are presented in Additional file 1. 


\section{Tissue segmentation on IHC WSIs}

A convolutional neural network $(\mathrm{CNN})$ was trained for tissue classification of CD3 and CD8 stained WSIs. First, we used the HE tiles dataset to train a VGG-19 model $(\mathrm{CNN}-0)$ with random initialization. Then we fine-tuned the trained model (CNN-HE) by transfer learning with the IHC tiles training dataset to generate a CNN-IHC model. Finally, the CNN-IHC model's classification performance was evaluated using the IHC tiles test dataset (Fig. 1A). Specifically, at the pre-train stage, the HE tiles dataset (283 k) was used to train a VGG-19 model for HE tiles nine categories classification. $10 \%$ of the samples were randomly selected as an internal validation set to monitor the training process. The training procedure generally followed Simonyan et al. [14], except for setting the batch size to 64 . At the transfer learning stage, the IHC tiles training dataset (154.4 $\mathrm{k}$ tiles) served as the training set. Fine-tuning was used to train the CNN-HE model with SGDM. The mini-batch size was set as 64 , and a fixed learning rate of $3 \times 10^{-4}$ was used to train the model for ten epochs, generating a CNN-IHC model. We trained the network on a desktop workstation with one NVIDIA GeForce RTX 2080Ti GPU. The CNN-IHC model training and testing were done using in MATLAB environment (R2020a, MathWorks, USA). The trained CNN-IHC model is available online (https://doi.org/10. 5281/zenodo.5589269).

In the rough segmentation step, the CD3 and CD8 stained WSIs were scaled to $20 \times$ magnification. Then overlapped tiles (224 pixels $\times 224$ pixels) were extracted from WSIs with a 75-pixel overlapped border. The CNNIHC model classified the cropped tile as one tissue type with the maximum probability (Fig. 1B).

\section{Stroma-immune score}

The stroma region segmentation result was mapping on the CD3 and CD8 WSIs as the region of interest (ROI). Then the positive $\mathrm{CD} 3 / \mathrm{CD} 8 \mathrm{~T}$-cells $\left(\mathrm{CD}^{+} / \mathrm{CD}^{+}\right)$that presented as the brown color within the ROI were segmented and counted by an in-house program. The mean density of positive cells in the stroma region was calculated (Fig. 1C). The immune cell density of each patient was converted as the percentile value (range from 0 to $100 \%)$ according to the immune cell distribution in the development group.
Hence, CD3 and CD8 percentiles were obtained, and the average of these two percentiles was calculated to obtain a stroma-immune score (percentile). According to the stroma-immune score (percentile) distribution in the development group, patients were divided into three categories (high, intermediate, and low) to obtain the threecategory stroma-immune score. These two thresholds were determined in the development group to balance the proportion of patients in each category by using the cut2 function from the Hmisc R package [15]. Then the intermediate and high scores were combined into a new high score, forming a two-category score (low vs. high). All analysis steps would be tested in the internal and external validation groups.

\section{Evaluation of the stroma-immune score}

For the three- or two-category stroma-immune score, the Kaplan-Meier method was used to analyze the survival curves. The log-rank test was used to test the differences in survival distributions. The Cox proportional hazards model was used to compute the hazard ratio (HR) of the stroma-immune score and other clinicopathological risk factors (age, sex, TNM stage, and tumor site) for OS. Subgroup analyses were performed by age, sex, TNM stage, tumor site, MSI status, and treatment modalities in Cohort 1. The performance of stroma-immune score and other factors were assessed by Harrell's C-index with 1000 times bootstrap.

\section{Stroma-immune score and TSR}

We also calculated the TSR, which was defined as the proportion of stroma area in the sum area of tumor epithelium and stroma in the WSI. Patients were grouped as stroma-low and stroma-high using a fixed threshold of 50\%. In stroma-low and stroma-high subgroups, Kaplan-Meier curves were plotted for the three categories stroma-immune score in Cohort 1.

\section{Stroma-immune score and intraepithelial-immune score}

We further established an intraepithelial-immune score, which summarized the mean density of $\mathrm{CD}^{+}$and $\mathrm{CD}^{+}$T-cells immune infiltration in the intraepithelial tumor. The intraepithelial-immune score was calculated the same way as the stroma-immune score, except that the ROI was replaced by the tumor epithelium region

(See figure on next page.)

Fig. 1 Study workflow. A Convolutional neural networks for colorectal tissue classification. First, a HE tiles dataset was used to pre-train an untrained VGG-19 network (CNN-0) as the CNN-HE model. Next, an IHC tiles training dataset was used to train the CNN-HE model as the CNN-IHC model with transfer learning. An independent dataset was used to test the tissue tiles classification performance of the CNN-IHC model. B Rough segmentation of IHC (CD3 and CD8) WSI. The CNN-IHC model was used to perform patch-level segmentation of IHC WSI. C Immune cells in the stroma region. The segmented stroma region was mapped on the original WSI, and $\mathrm{CD}^{+}$and $\mathrm{CD} 8^{+} \mathrm{T}$-cells in this region were segmented and counted. HE hematoxylin-eosin, IHC immunohistochemical, WSI whole-slide image, ADI adipose, BAC background, DEB debris, LYM lymphocytes, MUC mucus, MUS muscle, NOR normal mucosa, STR stroma, TUM tumor epithelium 
A
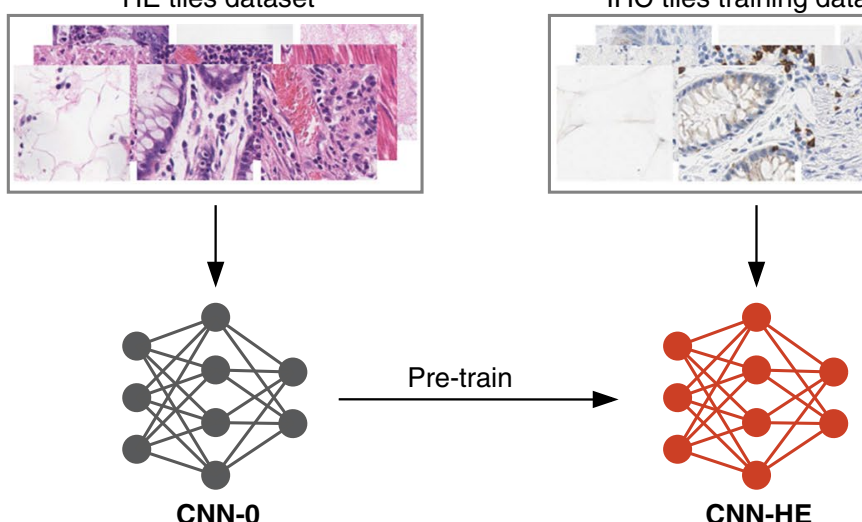

CNN-HE

$\mathrm{IHC}$ tiles training dataset
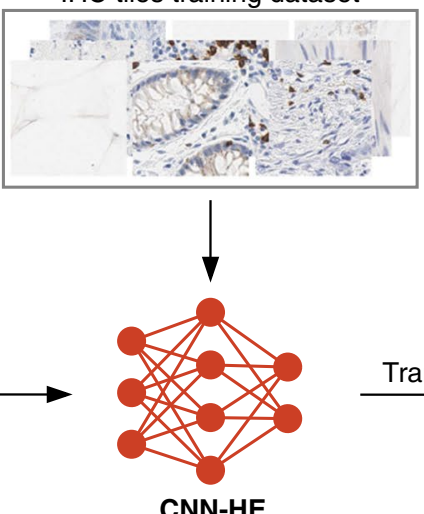
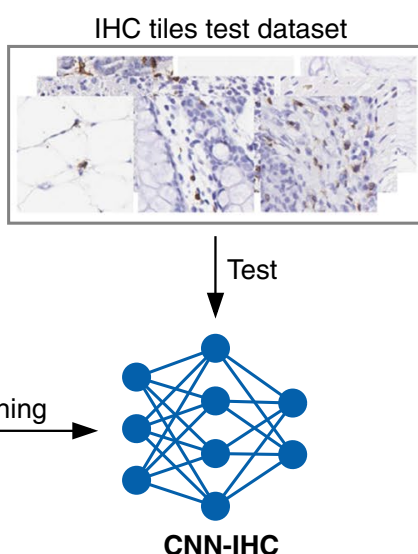

B

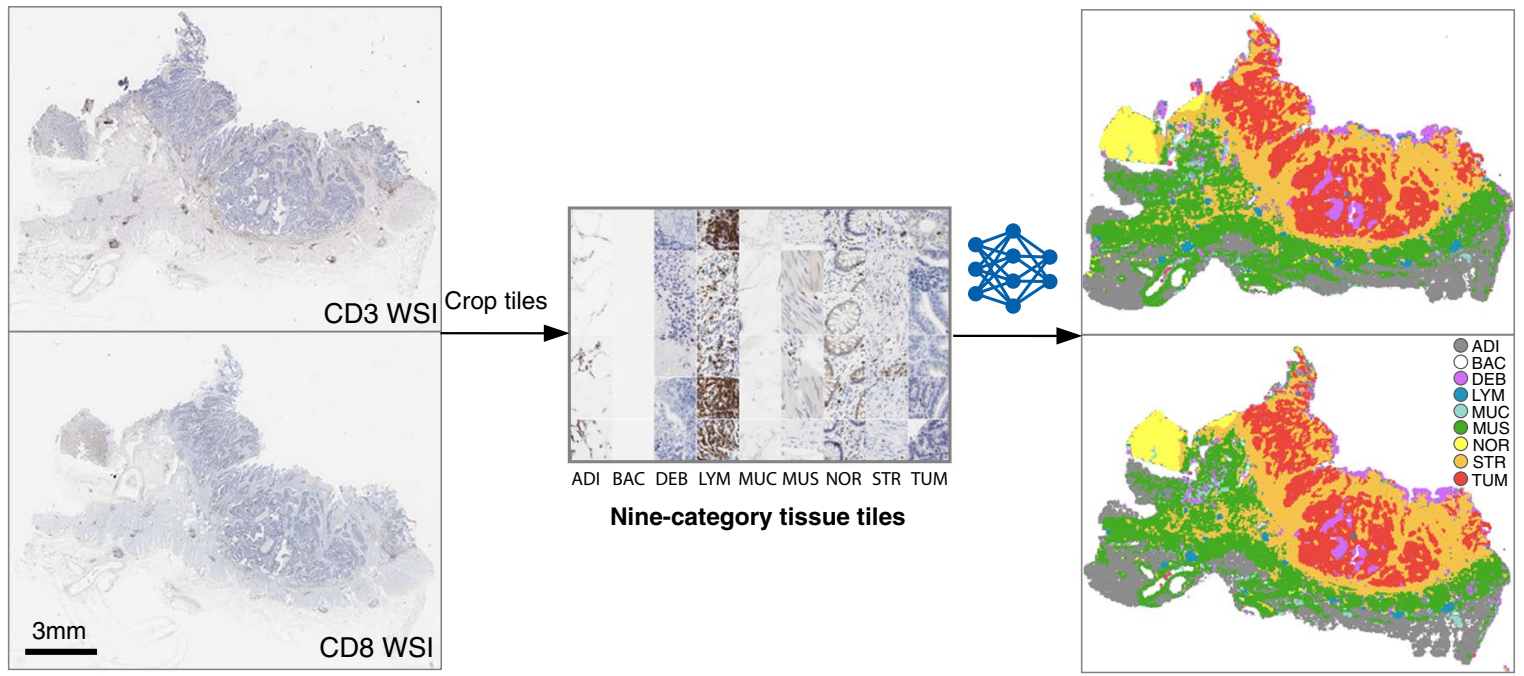

C

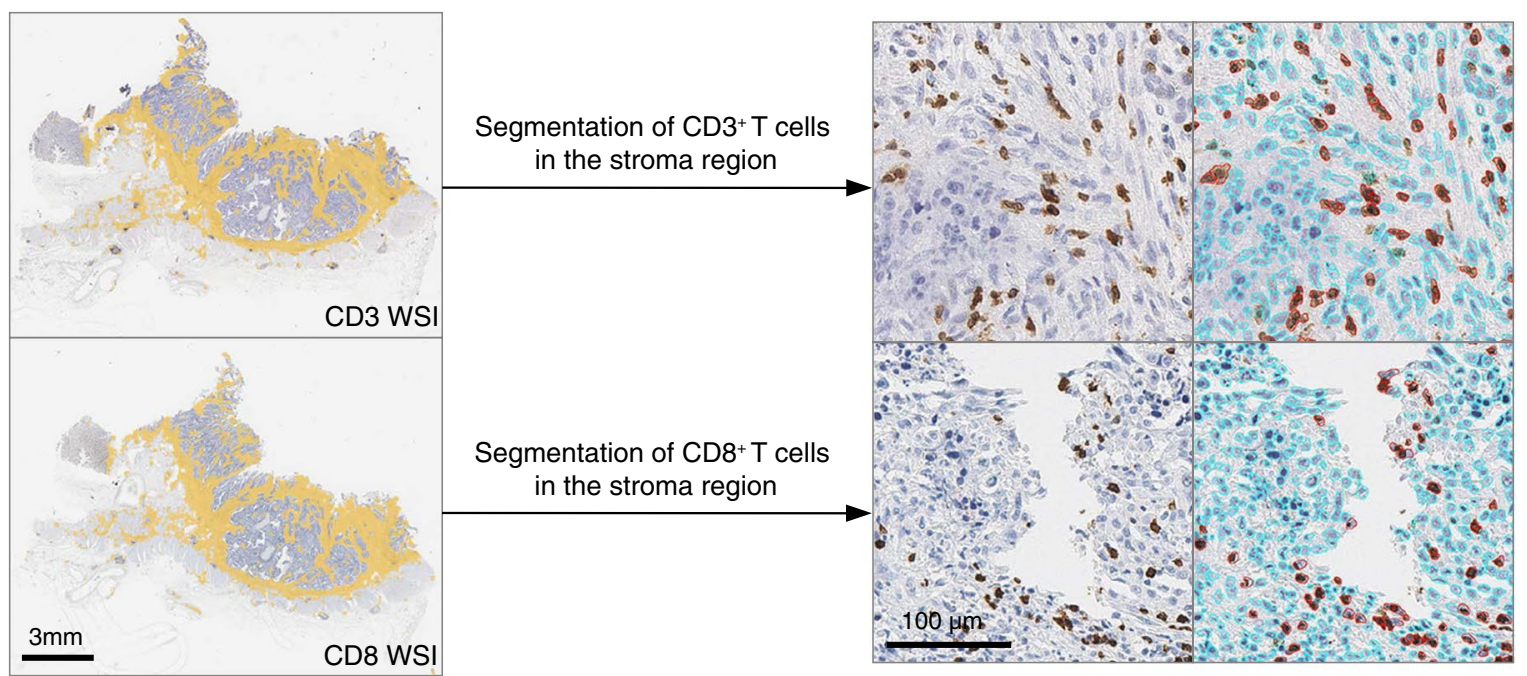

Fig. 1 (See legend on previous page.) 
in Cohort 1. The Pearson correlation coefficient [16] of the stroma-immune score and intraepithelial-immune score was calculated, and multivariate analysis was also performed.

\section{Statistical analysis}

All statistical analyses were performed with $\mathrm{R}$ software (version 3.6.1) [17]. Clinicopathological characteristics were compared among the three groups by Kruskal-Wallis rank sum test or Chi-square test when appropriate. Multiple comparisons correction was applied by Bonferroni correction. Two-sided $\mathrm{P}<0.05$ was considered statistically significant.

\section{Results \\ Patients}

A total of 424 patients were included in our study (Additional file 1: Fig. S1). The development group included 200 patients (aged $63.55 \pm 11.21$ years), the internal validation group included 134 patients $(62.78 \pm 13.31$ years), and the external validation group included 90 patients (62.34 \pm 12.74 years). In Cohort 1, 147 (44.0\%) patients had stage II colorectal cancer and 187 (56.0\%) patients had stage III colorectal cancer. In Cohort 2, there were $45(50.0 \%)$ and $45(50.0 \%)$ patients with stage II and III rectal cancer, respectively. The median follow-up time was 76 months in Cohort 1 and 69.5 months in Cohort 2. The 5-year survival rate was $71.5 \%$ (95\% CI $66.9-76.5 \%$ ) in Cohort 1 and 82.0\% (74.4-90.4\%) in Cohort 2. There were no statistical significances between the three groups in terms of age, sex, T-category, N-category, and TNM stage (all P $>0.05$; Table 1), except for the tumor site. Perhaps because of the lack of colon cancer patients in the external validation group.

\section{Tissue classification performance}

High classification accuracy performance was achieved in all tissue classes by CNN-IHC model (IHC tiles training dataset: 0.988, 95\% confidence interval [CI] 0.987-0.989; IHC tiles test dataset: $0.973,95 \%$ CI $0.971-0.975)$, which could be observed from the confusion matrixes (Fig. 2A, B).

\section{Prognostic value of the stroma-immune score}

The $\mathrm{CD}^{+}$and $\mathrm{CD} 8^{+}$mean densities in the stroma region were presented in Fig. 2C. A moderate correlation was observed $(\mathrm{r}=0.58, \mathrm{P}<0.001)$ between $\mathrm{CD}^{+}$and $\mathrm{CD}^{+}$ T-cells mean densities (Fig. 2D). Patients were classified into low, intermediate, and high stroma-immune score groups based on $40 \%$ and $63.5 \%$ thresholds, splitting patients into three percentile groups in the development group.
Table 1 The distributions of demographic and clinicopathologic characteristics of colorectal cancer patients in the three groups

\begin{tabular}{lrlll}
\hline & $\begin{array}{l}\text { Development } \\
\text { group }\end{array}$ & $\begin{array}{l}\text { Internal } \\
\text { validation } \\
\text { group }\end{array}$ & $\begin{array}{l}\text { External } \\
\text { validation } \\
\text { group }\end{array}$ & P \\
\hline $\begin{array}{l}\text { Age (years) } \\
\text { Mean }\end{array}$ & 63.55 & 62.78 & 62.34 & $0.845^{\#}$ \\
SD & 11.21 & 13.31 & 12.74 & \\
Sex & & & & $0.134^{\# \#}$ \\
Male & $129(64.5 \%)$ & $62(53.7 \%)$ & $56(62.2 \%)$ & \\
Female & $71(35.5 \%)$ & $72(46.3 \%)$ & $34(37.8 \%)$ & \\
T-category & & & & $0.161^{\# \#}$ \\
T1 & $1(0.5 \%)$ & $0(0 \%)$ & $2(2.2 \%)$ & \\
T2 & $10(5.0 \%)$ & $4(3.0 \%)$ & $3(3.3 \%)$ & \\
T3 & $168(84.0 \%)$ & $119(88.8 \%)$ & $82(91.1 \%)$ & \\
T4 & $21(10.5 \%)$ & $11(8.2 \%)$ & $3(3.3 \%)$ & \\
N-category & & & & \\
N0 & $82(41.0 \%)$ & $64(47.8 \%)$ & $45(50 \%)$ & \\
N1 & $71(35.5 \%)$ & $46(34.3 \%)$ & $33(36.7 \%)$ & \\
N2 & $47(23.5 \%)$ & $24(17.9 \%)$ & $12(13.3 \%)$ & \\
Stage & & & & \\
II & $82(41.0 \%)$ & $65(48.5 \%)$ & $45(50 \%)$ & \\
III & $118(59.0 \%)$ & $69(51.5 \%)$ & $45(50 \%)$ & \\
Tumor site & & & $0.240^{\# \#}$ \\
Colon & $115(57.5 \%)$ & $73(54.5 \%)$ & $0(0 \%)$ & \\
Rectum & $85(42.5 \%)$ & $61(45.6 \%)$ & $90(100 \%)$ & \\
\hline
\end{tabular}

${ }^{\#}$ Kruskal-Wallis rank sum test

\#\# Chi-square test

Patients with higher stroma-immune scores had much longer survival. Five-year survival rates of low, intermediate, and high stroma-immune score in the development group were $55.7 \%, 75.4 \%$, and $86.2 \%$ (HR for high vs. low 0.30, 95\% CI 0.16-0.58, P <0.001; Fig. 3A; Table 2). These results were confirmed in the internal validation group: the survival rates at 5 years were $57.2 \%$ in the low group, $76.2 \%$ in the intermediate group, and $81.4 \%$ in the high group (HR for high vs. low 0.40, 95\% CI 0.19-0.85, $\mathrm{P}=0.017$; Fig. 3C), while no significant difference was found in external validation group (HR for high vs. low $0.40,95 \%$ CI 0.13-1.22, $\mathrm{P}=0.110$; Fig. 3E). When the stroma-immune score was classified into two categories, the intermediate and high scores were combined into a new high score, patients with the new high stroma-immune score still showed significant prolonged OS in the development group with 5-year survival rates of the low and high scores were $55.7 \%$ and $80.8 \%$ (HR for high vs. low 0.39 , 95\% CI 0.24-0.63, P <0.001; Fig. 3B; Table 3), and the results were further confirmed in the internal validation group with 5-year survival rates of the low and high score were $57.1 \%$ and $78.8 \%$ (HR for high vs. 


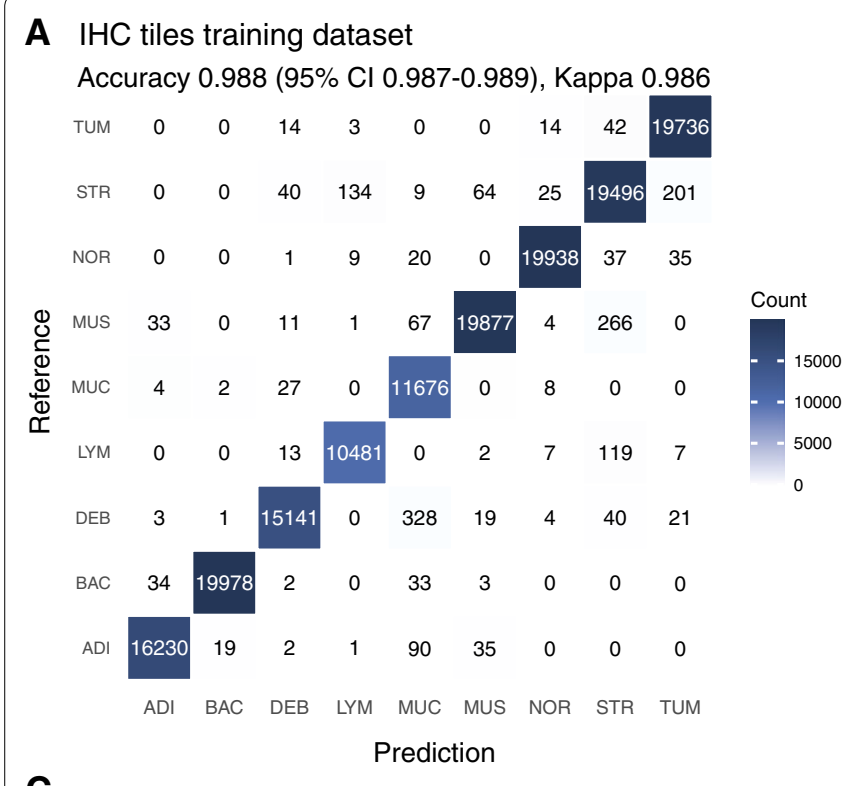

B IHC tiles test dataset

Accuracy 0.973 (95\% Cl 0.971-0.975), Kappa 0.970

$\mathbf{C}$
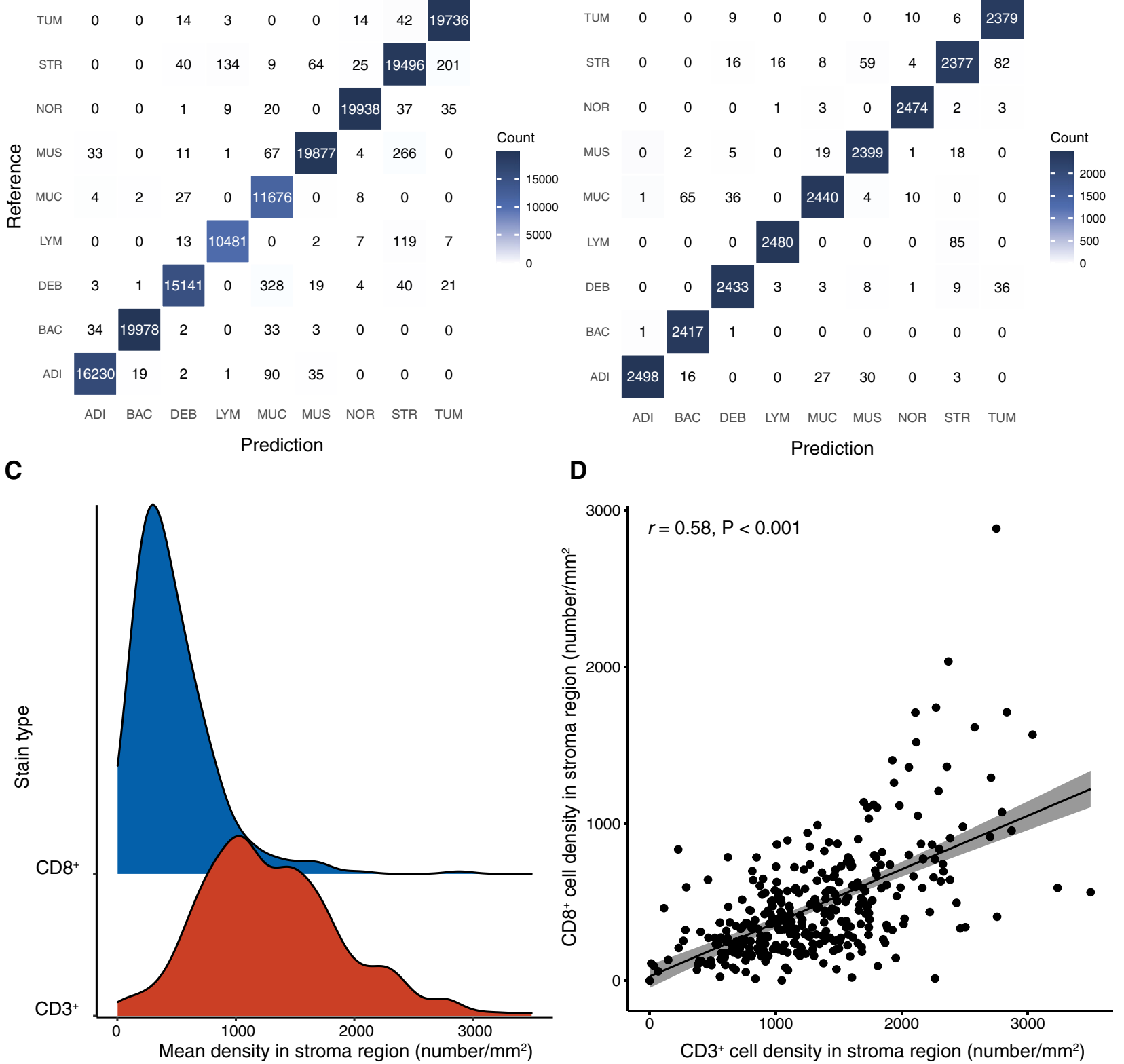

Fig. $2 \mathrm{IHC}$ tissue tiles classification performance on the IHC tiles training $(\mathbf{A})$ and test (B) sets. C Mean density distributions of the two cell counting results. $\mathbf{D}$ Correlation analysis between the $\mathrm{CD}^{+}$mean density and the $\mathrm{CD}^{+}$mean density in the stroma region. IHC immunohistochemical

low $0.49,95 \%$ CI $0.28-0.88, \mathrm{P}=0.017$; Fig. 3D), and external validation group with 5 -year survival rates of the low and high score were $63.9 \%$ and $88.9 \%$ (HR for high vs. low $0.35,95 \%$ CI $0.15-0.83, \mathrm{P}=0.018$; Fig. $3 \mathrm{~F}$ ).

By stratified analysis with TNM stage, age, sex, and tumor site, the stroma-immune score (3-category) remained a statistically significant predictor for OS at Cohort 1 (all $\mathrm{P}<0.05$; Additional file 1: Fig. S2). MSI status was available in 257 patients in Cohort 1, including 27 patients with MSI and 230 patients with MSS. When stroma-immune score stratified into two categories by combining intermediate and high groups into a new high group, patients with high stroma-immune score had prolonged OS in both MSI and MSS subgroup (MSI: unadjusted HR for high vs. low $0.11,95 \%$ CI 0.01-1.03, $\mathrm{P}=0.053$; and MSS: 0.50, 0.32-0.77, 0.002; Additional 
A Development group (3-category)

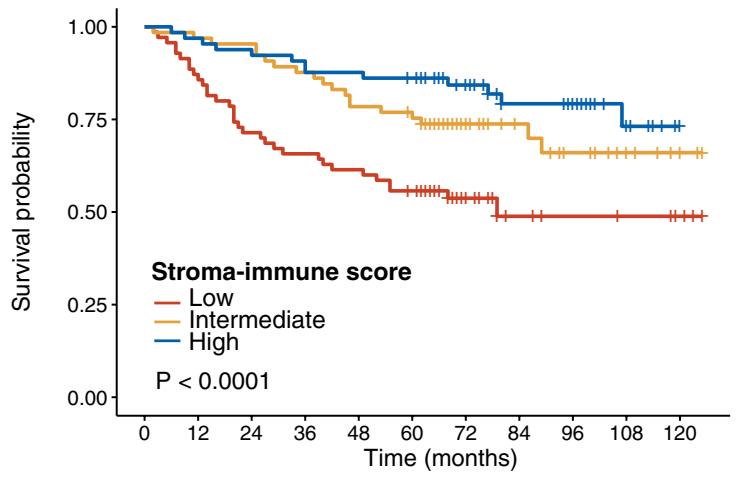

Number at risk

$\begin{array}{llllllllllll}\text { Low } & 70 & 61 & 50 & 46 & 43 & 35 & 21 & 8 & 6 & 5 & 3 \\ \text { Intermediate } & 65 & 63 & 62 & 57 & 51 & 49 & 31 & 19 & 13 & 8 & 4\end{array}$

$\begin{array}{llllllllllll}\text { High } & 65 & 63 & 61 & 59 & 57 & 55 & 44 & 29 & 25 & 12 & 1\end{array}$

C Internal validation group (3-category)

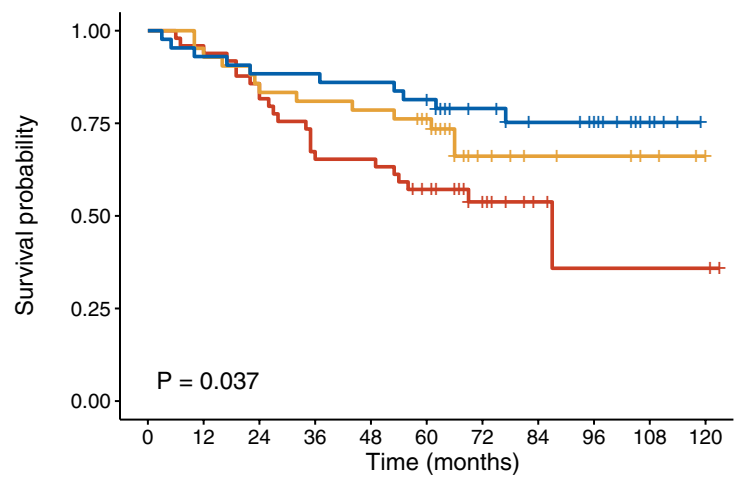

Number at risk

$\begin{array}{llllllllllll}\text { Low } & 49 & 47 & 42 & 33 & 32 & 24 & 13 & 4 & 2 & 2 & 2 \\ \text { Intermediate } & 42 & 40 & 36 & 34 & 33 & 29 & 11 & 7 & 6 & 3 & 1 \\ \text { High } & 43 & 40 & 38 & 38 & 37 & 35 & 23 & 18 & 15 & 5 & 0\end{array}$

E External validation group (3-category)

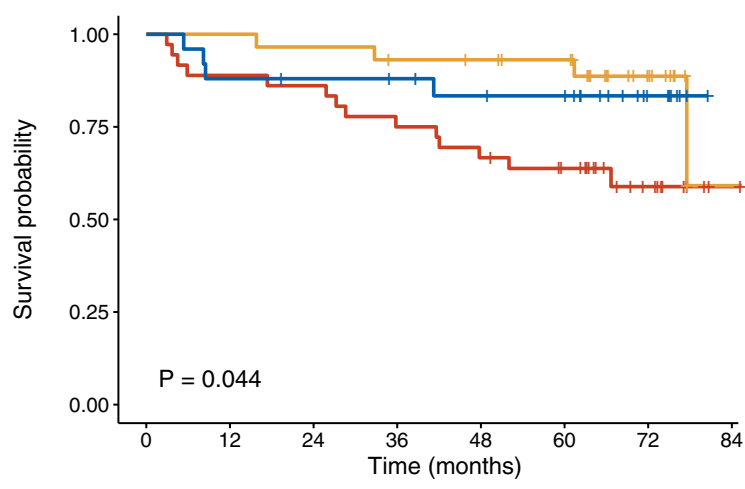

Number at risk
B Development group (2-category)

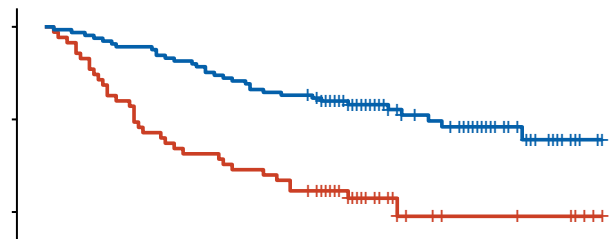

\section{Stroma-immune score}

- Low

- High

$P<0.0001$

$\begin{array}{llllllllll}0 & 12 & 24 & 36 & \begin{array}{c}1 \\ 0\end{array} & \begin{array}{c}10 \\ \text { Time (months) }\end{array} & \begin{array}{c}72 \\ 84\end{array} & 96 & 108 & 120\end{array}$

Number at risk

$\begin{array}{lccccccccccc}\text { Low } & 70 & 61 & 50 & 46 & 43 & 35 & 21 & 8 & 6 & 5 & 3 \\ \text { High } & 130 & 126 & 123 & 116 & 108 & 104 & 75 & 48 & 38 & 20 & 5\end{array}$

D Internal validation group (2-category)

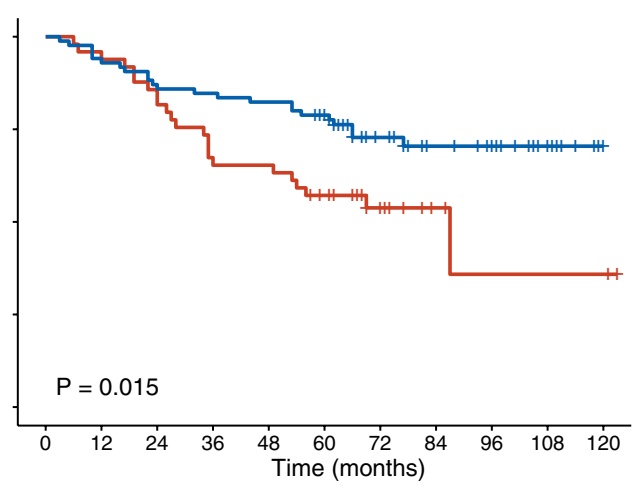

Number at risk

Low $\begin{array}{lllllllllll}49 & 47 & 42 & 33 & 32 & 24 & 13 & 4 & 2 & 2 & 2\end{array}$ $\begin{array}{lllllllllll}85 & 80 & 74 & 72 & 70 & 64 & 34 & 25 & 21 & 8 & 1\end{array}$

\section{F External validation group (2-category)}

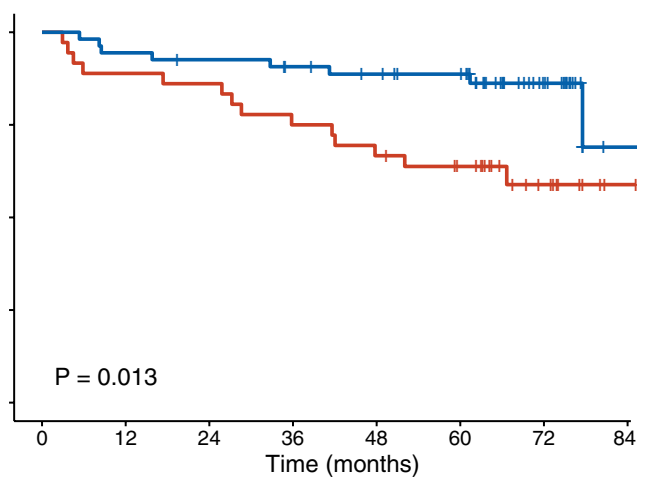

Number at risk

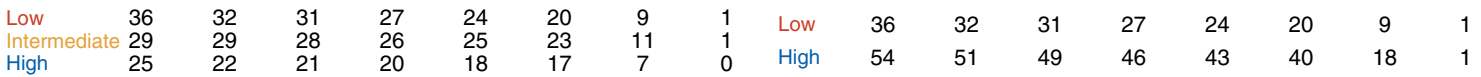

Fig. 3 Kaplan-Meier curves analysis for the stroma-immune score in development, internal and external validation group. A, C, E Stroma-immune score (3-category); B, D, F Stroma-immune score (2-category, the intermediate and high score were combined into a new high score) 
Table 2 Unadjusted and multivariate analyses for overall survival (3-category stroma-immune score)

\begin{tabular}{|c|c|c|c|c|c|c|c|c|c|}
\hline & \multicolumn{3}{|c|}{ Development group } & \multicolumn{3}{|c|}{ Internal validation group } & \multicolumn{3}{|c|}{ External validation group } \\
\hline & HR & $95 \% \mathrm{Cl}$ & $\mathbf{P}$ & HR & $95 \% \mathrm{Cl}$ & $\mathbf{P}$ & HR & $95 \% \mathrm{Cl}$ & $\mathbf{P}$ \\
\hline \multicolumn{10}{|c|}{ Unadjusted stratified Cox model } \\
\hline \multicolumn{10}{|c|}{ Stroma-immune score } \\
\hline Low & 1 & & & 1 & & & 1 & & \\
\hline Intermediate & 0.48 & $0.27-0.84$ & 0.011 & 0.60 & $0.30-1.18$ & 0.140 & 0.31 & $0.10-0.94$ & 0.039 \\
\hline High & 0.30 & $0.16-0.58$ & $<0.001$ & 0.40 & $0.19-0.85$ & 0.017 & 0.40 & $0.13-1.22$ & 0.110 \\
\hline \multicolumn{10}{|c|}{ Multivariable stratified Cox model } \\
\hline \multicolumn{10}{|c|}{ Stroma-immune score } \\
\hline Low & 1 & & & 1 & & & 1 & & \\
\hline Intermediate & 0.45 & $0.25-0.79$ & 0.006 & 0.60 & $0.30-1.19$ & 0.15 & 0.34 & $0.11-1.06$ & 0.062 \\
\hline High & 0.35 & $0.18-0.66$ & 0.001 & 0.43 & $0.20-0.92$ & 0.029 & 0.40 & $0.13-1.23$ & 0.110 \\
\hline \multicolumn{10}{|l|}{ TNM stage } \\
\hline$\|$ & 1 & & & 1 & & & 1 & & \\
\hline III & 3.25 & $1.76-6.00$ & $<0.001$ & 2.63 & $1.40-4.95$ & 0.003 & 1.77 & $0.75-4.20$ & 0.195 \\
\hline Age & 1.04 & $1.01-1.06$ & 0.007 & 1.02 & $0.99-1.04$ & 0.15 & 1.03 & $1.00-1.07$ & 0.072 \\
\hline
\end{tabular}

HR hazard ratio, Cl confidence interval, TNM tumor-node-metastasis

file 1: Fig. S3). Treatment modalities were available in 130 patients in the whole cohort: 47 were treated with surgery alone, and 83 with surgery and adjuvant chemotherapy. There was no significant difference in the stroma-immune score (2-category) for OS in both treatment groups ( $\mathrm{P}>0.05$; Additional file 1: Fig. $\mathrm{S} 4)$.

In the multivariate analysis, the TNM stage, age, and stroma-immune score (2-category) were identified as independent predictors for OS, patients with a high stroma-immune score associated with better OS in the development group (HR for high vs. low 0.40, 95\% CI 0.24-0.66, $\mathrm{P}<0.001$ ), internal validation group (HR for high vs. low $0.52,95 \%$ CI $0.29-0.93, \mathrm{P}=0.027$ ), and external validation group (HR for high vs. low $0.37,95 \%$ CI 0.15-0.89, $\mathrm{P}=0.027$; Table 3). Additional file 1: Fig. S5 shows how the pipeline can be used to predict the prognosis of one patient with CD3 and CD8 WSIs.

\section{Stroma-immune score and TSR}

For Cohort 1, patients with stroma-high had worse OS (HR for stroma-high vs. stroma-low 1.48, 95\% CI 1.01-2.19, $\mathrm{P}=0.046$; Additional file 1: Fig. S6). In the stroma-low group, the stroma-immune score still has a prognostic value, wherein patients with high stromaimmune scores had the best outcome (Fig. 4A). In the stroma-high group, patients with low stroma-immune

Table 3 Unadjusted and multivariate analyses for overall survival (2-category stroma-immune score)

\begin{tabular}{|c|c|c|c|c|c|c|c|c|c|}
\hline & \multicolumn{3}{|c|}{ Development group } & \multicolumn{3}{|c|}{ Internal validation group } & \multicolumn{3}{|c|}{ External validation group } \\
\hline & HR & $95 \% \mathrm{Cl}$ & $\mathbf{P}$ & HR & $95 \% \mathrm{Cl}$ & $\mathbf{P}$ & HR & $95 \% \mathrm{Cl}$ & $\mathbf{P}$ \\
\hline \multicolumn{10}{|c|}{ Unadjusted stratified Cox model } \\
\hline \multicolumn{10}{|c|}{ Stroma-immune score } \\
\hline Low & 1 & & & 1 & & & 1 & & \\
\hline High & 0.39 & $0.24-0.63$ & $<0.001$ & 0.49 & $0.28-0.88$ & 0.017 & 0.35 & $0.15-0.83$ & 0.018 \\
\hline \multicolumn{10}{|c|}{ Multivariable stratified Cox model } \\
\hline \multicolumn{10}{|c|}{ Stroma-immune score } \\
\hline Low & 1 & & & 1 & & & 1 & & \\
\hline High & 0.40 & $0.24-0.66$ & $<0.001$ & 0.52 & $0.29-0.93$ & 0.027 & 0.37 & $0.15-0.89$ & 0.027 \\
\hline \multicolumn{10}{|c|}{ TNM stage } \\
\hline$\|$ & 1 & & & 1 & & & 1 & & \\
\hline III & 3.31 & $1.80-6.09$ & $<0.001$ & 2.68 & $1.42-5.04$ & 0.002 & 1.79 & $0.76-4.22$ & 0.184 \\
\hline Age & 1.04 & $1.01-1.06$ & 0.006 & 1.02 & $0.99-1.04$ & 0.151 & 1.03 & $1.00-1.07$ & 0.072 \\
\hline
\end{tabular}

$H R$ hazard ratio, Cl confidence interval, TNM tumor-node-metastasis 


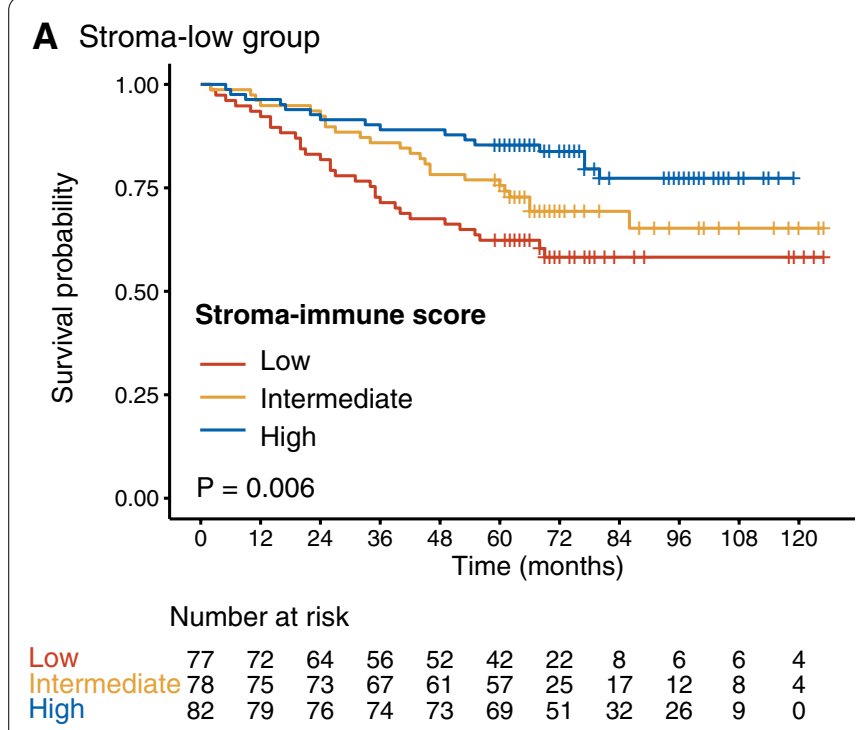

B Stroma-high group

C

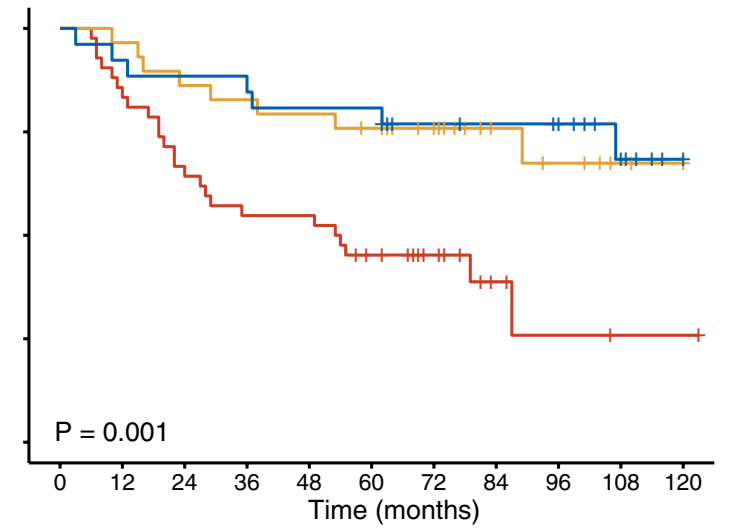

Number at risk

$\begin{array}{lllllllcccc}42 & 36 & 28 & 23 & 23 & 17 & 12 & 4 & 2 & 1 & 1 \\ 29 & 28 & 25 & 24 & 23 & 21 & 17 & 9 & 7 & 3 & 1 \\ 26 & 24 & 23 & 23 & 21 & 21 & 16 & 15 & 14 & 8 & 1\end{array}$

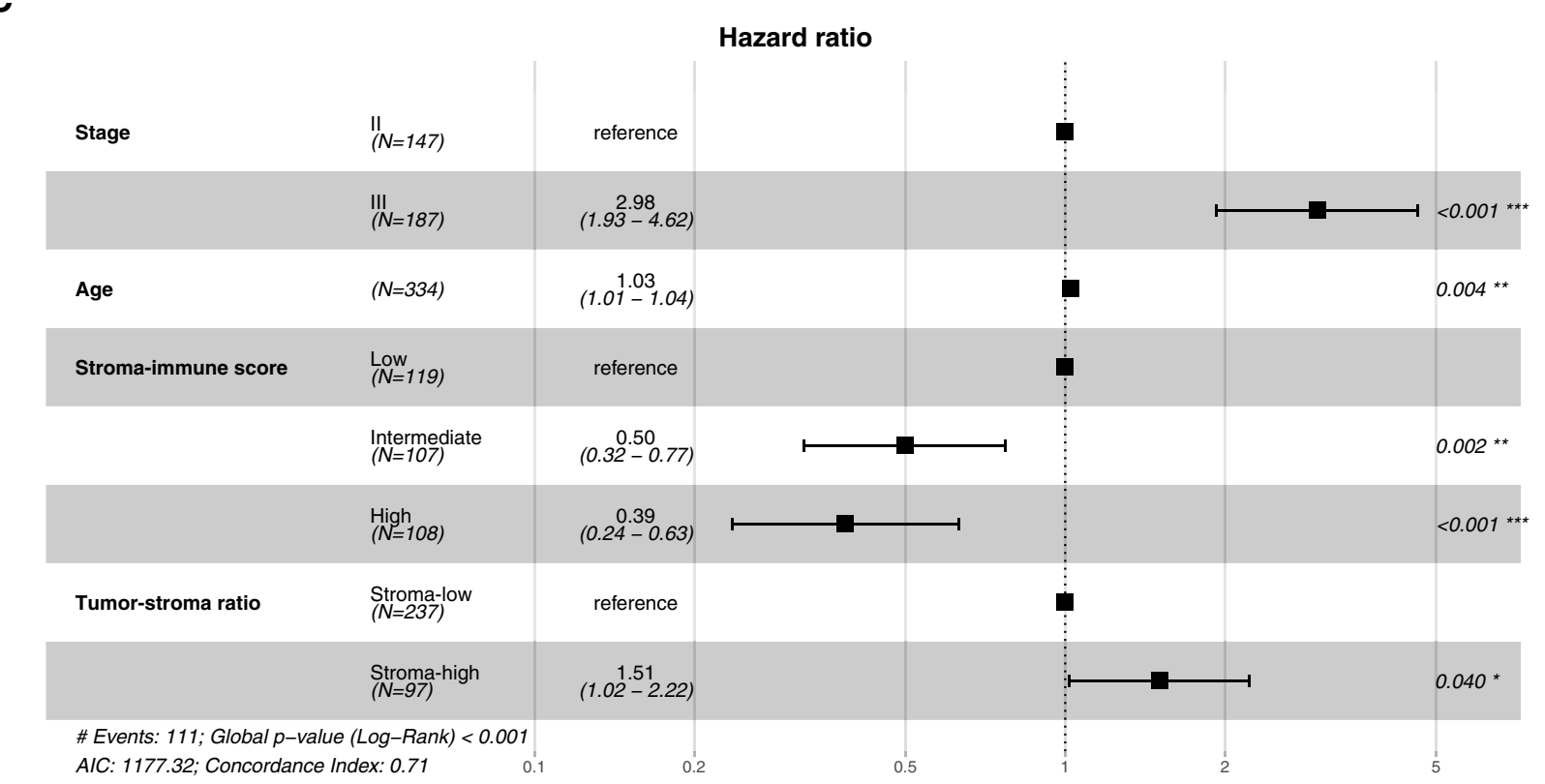

Fig. 4 A Kaplan-Meier survival curves for the stroma-immune score in the stroma-low group. B Kaplan-Meier survival curves for the stroma-immune score in the stroma-high group. C HRs with 95\% Cl in the multivariable Cox model, including TNM stage (II-III), sex, age, stroma-immune score, and stroma-tumor ratio. HR hazard ratio, $\mathrm{Cl}$ confidence interval

scores had the worst survival (Fig. 4B). When we performed the multivariate analysis in Cohort 1, we found that the stroma-immune score was independent of TSR (Fig. 4C).

\section{Stroma-immune score and intraepithelial-immune score}

We found that for both CD3 and CD8, intraepithelial immune cell density was highly correlated with stromal immune cell density in Cohort 1 (Additional file 1: Fig. S7A, B). For stroma-immune score (percentile) and intraepithelial-immune score (percentile), a strong correlation was observed $(\mathrm{r}=0.70, \mathrm{P}<0.001$; Additional file 1: Fig. S7C). Patients were classified as high, intermediate, and low intraepithelial-immune scores by $37.0 \%$ and $64.5 \%$ thresholds. Patients with high intraepithelialimmune scores had prolonged OS (HR for high vs. low $0.36,95 \%$ CI $0.22-0.61, \mathrm{P}<0.001$ ) at Cohort 1 . We found that the intraepithelial-immune score was not independent of the stroma-immune score in multivariate analysis (Additional file 1: Fig. S7D). 
The added prognostic value of the stroma-immune score We obtained C-index distributions of TNM stage, stroma-immune score, and TNM stage plus stromaimmune score in the development, internal and external validation groups with the bootstrap method. In the development group, the combination of the TNM stage and the stroma-immune score showed better discrimination power than the TNM stage alone (mean C-index: 0.70 vs. $0.62, \mathrm{P}<0.001$ after Bonferroni correction; Fig. 5A). The result was confirmed in the internal validation group (0.67 vs. $0.64, \mathrm{P}<0.001$ after Bonferroni correction; Fig. $5 \mathrm{~B})$ and external validation group (0.65 vs. $0.58, \mathrm{P}<0.001$ after Bonferroni correction; Fig. 5C).

\section{Discussion}

To quantify the immune infiltration within the stroma region in patients with II-III CRC, we designed a deep learning-based pipeline for $\mathrm{CD}^{+}$and $\mathrm{CD} 8^{+} \mathrm{T}$-cells calculation on IHC-stained WSIs. A scoring system, named stroma-immune score, was established. Its added prognostic value was evaluated and validated in development, internal, and external validation groups.

The prognosis value of immune infiltrates quantification in CRC has been supported by mounting evidence $[5,7,18,19]$. Especially, immune infiltration of $\mathrm{CD}^{+}$ and $\mathrm{CD}^{+}$T-cells subsets in tumor regions has been widely confirmed to be associated with prognosis. CD3 is a common membrane marker for mature $\mathrm{T}$ lymphocytes and can be used to represent total $\mathrm{T}$ lymphocytes. $\mathrm{CD}^{+}$cytotoxic $\mathrm{T}$ cells are the main antitumor $\mathrm{T}$ lymphocyte subsets [20]. Previous studies have shown that remodeling of the extracellular matrix, also known as the stroma, can act as a physical barrier, limiting immune cells' access to cancer cells [21-24]. Increased tumorstromal composition and decreased intratumoral infiltrating lymphocytes are associated with poor overall survival [24]. A recent study by Reichiling et al. suggests that the prognosis evaluation of CRC acquires further insight into the stromal immune infiltration $\left(\mathrm{CD}^{+}\right.$and $\mathrm{CD} 8^{+} \mathrm{T}$-cells) in addition to intrinsic tumor variables [25]. Moreover, Yoo et al. quantified intraepithelial TILs and stromal TILs separately to describe the landscape of the tumor-immune microenvironment [7]. We developed a deep learning-based stroma-immune score that takes $\mathrm{CD}^{+}$and $\mathrm{CD}^{+}{ }^{+}$T-cells in the stroma region into account to reveal patient prognosis in CRC. Our study showed that the fully automated quantified stromaimmune score enables prognostic stratification for stage II-III CRC, corroborating the significant role of stromal immune infiltration in the tumor-immune microenvironment. In addition, the stroma-immune score remained a statistically significant predictor for OS when stratified by TNM stage, age, sex, and tumor site, and MSI status, except for the treatment modalities. Despite no significant OS difference was found for the stroma-immune score in both adjuvant chemotherapy and surgery-only groups, we could observe the positive trends. However, further studies are still needed to explore whether the stroma-immune score can help assess patients' benefit from different treatment modalities. On the other hand, it is noteworthy that our proposed stroma-immune score pipeline could be more easily translated into routine clinical use regarding its reproducibility and reliability compared to subjective evaluation.

Since the TSR has been proven to be an independent prognostic factor for patients with CRC [10, 26], we also investigated the relationship between stromaimmune score and TSR. We found that the stromaimmune score was independent of TSR in multivariate analysis, and patients with stroma-low and high stromaimmune scores had the most favorable survival. In contrast, patients with stroma-high and low stroma-immune scores had the worst survival. These results were consistent with previous studies that patients with stroma-low
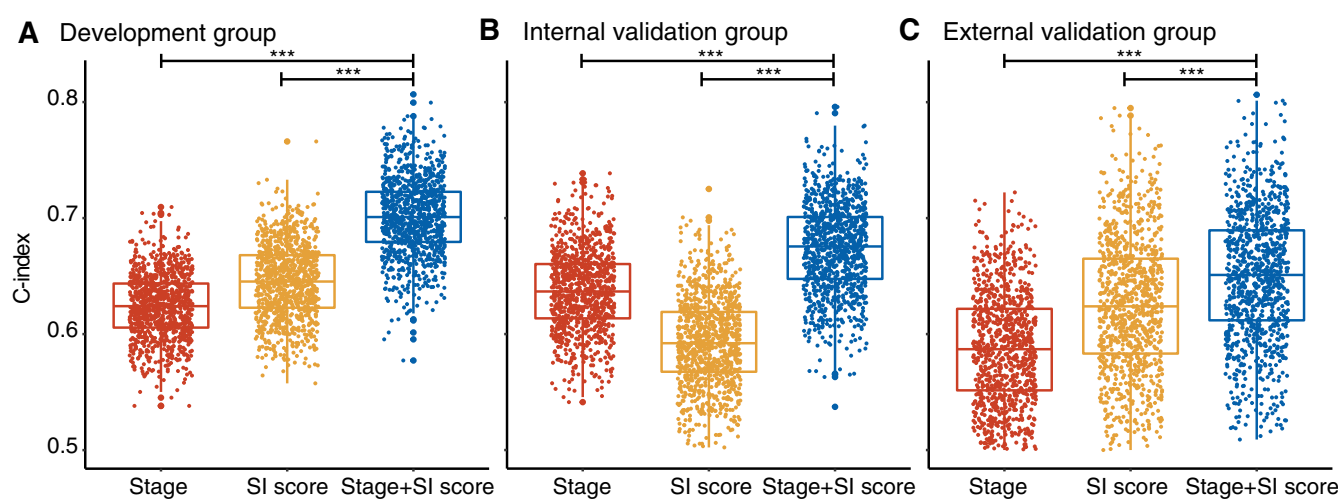

Fig. 5 The added prognostic value of stroma-immune score using a $1000 \times$ bootstrap strategy ${ }^{* * *} \mathrm{P}<0.001$ after Bonferroni correction). A Development group. B Internal validation group. C External validation group. S/ score stroma-immune score 
were associated with a higher survival rate, and patients with high TILs tend to have a better outcome $[7,10,26]$. Additionally, the $\mathrm{CD}^{+}$and $\mathrm{CD}^{+}{ }^{+} \mathrm{T}$-cells within the tumor epithelium region were also analyzed in our study. We observed that the stroma-immune score was strongly correlated with the intraepithelial-immune score. In addition, the stroma-immune score showed superior prognostic value compared with the intraepithelialimmune score. This result supported the idea of focusing on the immune infiltrates in the stroma and also demonstrated that our proposed stroma-immune score may be sufficient for OS prediction in patients with stage II-III CRC.

The tumor microenvironment characteristics have been shown to allow further insight into patients' prognosis in most solid tumors, including CRC [27, 28]. Unlike the Immunoscore [5], which quantifies $\mathrm{CD}^{+}$and $\mathrm{CD}^{+}$T-cells at the tumor core and the invasive margin, our proposed scoring strategy specifically focuses on the stroma region for these two types of immune cells density. As far as we know, Immunoscore takes the tumor region as a whole, ignoring the tumor microenvironment information of specific tissue types, such as immune expression in the stroma region, might be insufficient to capture the biological complexity. Additionally, even in the tumor core region, the stroma also serves an important role in the tumor microenvironment [29, 30]. We also observed that the invasive margin belonged to our automated segmented stroma region (Fig. 1C). Besides, most TILs were located in the stroma rather than tumor epithelium (mean density [cells $/ \mathrm{mm}^{2}$ ] of $\mathrm{CD}^{+}$T-cell: 1304 vs. $355 ; \mathrm{CD}^{+}: 472$ vs. 141$)$. Therefore, our study considered only the $\mathrm{CD}^{+}$and $\mathrm{CD}^{+}$T-cell in the stroma region to develop the stroma-immune score. As expected, our results showed that the stromaimmune score demonstrated higher discrimination performance for OS prediction compared with the previous study (C-index 0.63 vs. 0.58). However, additional studies should be performed to compare the stroma-immune score with Immunoscore directly.

The study has limitations. First, limited sample sizes were used to evaluate the prognostic value of the stroma-immune score, especially for the external validation group. Second, the presented results still require further prospective and widespread validation. Furthermore, considering the complexity of the tumor immune microenvironment, the prognostic values of other intratumoral-infiltrating T-cell subgroups, such as $\mathrm{CD}^{+}{ }^{+} \mathrm{FOXP}^{+}{ }^{+} \mathrm{T}$-cells, are needed for further exploring. Moreover, the prognostic value of the spatial distribution of immune invasion in tumor stroma or other tissue types is also one of the research directions we are interested in.
In conclusion, with deep learning, we built a fully automated pipeline to quantify $\mathrm{CD}^{+}$and $\mathrm{CD}^{+}{ }^{+}$T-cells densities in the stroma region on IHC-stained WSIs of stage II-III CRC. A stroma-immune score was calculated via digital pathology image analysis. We further used two groups to validate the prognostic value of stromaimmune score for OS. The stroma-immune score we proposed could be easily translated into routine pathologic assessment regarding its reproducibility and reliability.

\section{Supplementary Information}

The online version contains supplementary material available at https://doi. org/10.1186/s12935-021-02297-w.

Additional file 1: Figure S1. Study profile and IHC tiles dataset generation. Figure S2-S4. Kaplan-Meier survival analysis for patients stratified by TNM stage, age, sex, tumor site, MSI status, and treatment modalities. Figure S5. Using the pipeline to predict the prognosis of one patient with CD3 and CD8 WSIs. Figure S6. Kaplan-Meier survival curve of overall survival of stroma-low vs. stroma-high groups. Figure S7. Stroma-immune score vs. intraepithelial-immune score.

\section{Acknowledgements}

Not applicable.

\section{Authors' contributions}

Study concepts: ZaL, KZ, ZX, YL; Study design: ZX, KZ, SY, YW; Data acquisition: $Z X, S Y, Y W, Y H, Y M, S Z, Z a L ;$ Quality control of data and algorithms: $K Z, C H, X P$, ZS; Data analysis and interpretation: SY, XH, HL, XC; Statistical analysis: YX, CH; Manuscript preparation: ZX, SY; Manuscript editing: KZ, ZaL, YH, ZhL; Manuscript review: ZaL, QZ, CL. All authors read and approved the final manuscript.

\section{Funding}

This work was supported by the Key R\&D Program of Guangdong Province, China [2021B0101420006], the National Key Research and Development Program of China [2017YFC1309100], National Science Fund for Distinguished Young Scholars [81925023], National Natural Science Foundation of China [81771912, 82071892, 82072090, 62002082], and High-level Hospital Construction Project [DFJH201805].

\section{Availability of data and materials}

The datasets used and/or analyzed during the current study are available from the corresponding author on reasonable request.

\section{Declarations}

Ethics approval and consent to participate

This study was approved by the Research Ethics Committee of Guangdong Provincial People's Hospital (GDREC2020011H) and the institutional review board of the Sixth Affiliated Hospital of Sun Yat-sen University (2019ZSLYEC-169), and the informed consent was waived. This study was performed in accordance with the Declaration of Helsinki Ethical.

\section{Consent for publication}

Not applicable.

\section{Competing interests}

The authors declare that they have no competing interests.

\section{Author details}

'Department of Radiology, Guangdong Provincial People's Hospital, Guangdong Academy of Medical Sciences, 106 Zhongshan Er Road, Guangzhou 510080, China. ${ }^{2}$ School of Medicine, South China University of Technology, Panyu District, Guangzhou 510006, China. ${ }^{3}$ Department of General 
Surgery, Guangdong Provincial People's Hospital, Guangdong Academy of Medical Sciences, Guangzhou 510080, China. ${ }^{4}$ Department of Radiology, Zhuhai People's Hospital, Zhuhai Hospital Affiliated with Jinan University, Zhuhai 519000, China. ${ }^{5}$ Department of Pathology, The Sixth Affiliated Hospital of Sun Yat-Sen University, Guangzhou 510655, China. ${ }^{6}$ Department of Pathology, Guangdong Provincial People's Hospital, Guangdong Academy of Medical Sciences, Guangzhou 510080, China. ${ }^{7}$ Department of Radiology, The First Affiliated Hospital of Chongqing Medical University, Chongqing 400016, China. ${ }^{8}$ School of Bioengineering, Chongqing University, Chongqing 400044, China. ${ }^{9}$ The Second School of Clinical Medicine, Southern Medical University, Guangzhou 510080, China. ${ }^{10}$ Department of Radiology, Guangzhou First People's Hospital, Guangzhou 510180, China.

Received: 7 July 2021 Accepted: 23 October 2021

Published online: 30 October 2021

\section{References}

1. Bray F, Ferlay J, Soerjomataram I, Siegel RL, Torre LA, Jemal A. Global cancer statistics 2018: GLOBOCAN estimates of incidence and mortality worldwide for 36 cancers in 185 countries. CA Cancer J Clin. 2018;68:394-424.

2. Edge SB, Compton CC. The American Joint Committee on Cancer: the 7th edition of the AJCC cancer staging manual and the future of TNM. Ann Surg Oncol. 2010;17:1471-4.

3. Nagtegaal ID, Quirke P, Schmoll H-J. Has the new TNM classification for colorectal cancer improved care? Nat Rev Clin Oncol. 2012;9:119-23.

4. Dekker E, Tanis PJ, Vleugels JLA, Kasi PM, Wallace MB. Colorectal cancer. Lancet. 2019;394:1467-80.

5. Pagès F, Mlecnik B, Marliot F, Bindea G, Ou F-S, Bifulco C, et al. International validation of the consensus Immunoscore for the classification of colon cancer: a prognostic and accuracy study. Lancet. 2018;391:2128-39.

6. Trabelsi M, Farah F, Zouari B, Jaafoura MH, Kharrat M. An Immunoscore system based on CD3+ and CD8+ infiltrating lymphocytes densities to predict the outcome of patients with colorectal adenocarcinoma. OTT. 2019;12:8663-73.

7. Yoo S-Y, Park HE, Kim JH, Wen X, Jeong S, Cho N-Y, et al. Whole-slide image analysis reveals quantitative landscape of tumor-immune microenvironment in colorectal cancers. Clin Cancer Res. 2020;26:870-81.

8. Kather JN, Krisam J, Charoentong P, Luedde T, Herpel E, Weis C-A, et al Predicting survival from colorectal cancer histology slides using deep learning: a retrospective multicenter study. PLoS Med. 2019;16:e1002730.

9. Martin B, Banner BM, Schäfer E-M, Mayr P, Anthuber M, Schenkirsch G, et al. Tumor proportion in colon cancer: results from a semiautomatic image analysis approach. Virchows Arch. 2020;477:185-93.

10. Zhao K, Li Z, Yao S, Wang Y, Wu X, Xu Z, et al. Artificial intelligence quantified tumour-stroma ratio is an independent predictor for overall survival in resectable colorectal cancer. EBioMedicine. 2020;61:103054.

11. Bera K, Schalper KA, Rimm DL, Velcheti V, Madabhushi A. Artificial intelligence in digital pathology — new tools for diagnosis and precision oncology. Nat Rev Clin Oncol. 2019;16:703-15.

12. Nearchou IP, Lillard K, Gavriel CG, Ueno H, Harrison DJ, Caie PD. Automated analysis of lymphocytic infiltration, tumor budding, and their spatial relationship improves prognostic accuracy in colorectal cancer. Cancer Immunol Res. 2019;7:609-20.

13. Nearchou IP, Gwyther BM, Georgiakakis ECT, Gavriel CG, Lillard K, Kajiwara $Y$, et al. Spatial immune profiling of the colorectal tumor microenvironment predicts good outcome in stage II patients. npj Digit Med. 2020;3:71.

14. Simonyan K, Zisserman A. Very deep convolutional networks for largescale image recognition. arXiv:14091556 [cs]. 2015. http://arxiv.org/abs/ 1409.1556. Accessed 19 Nov 2020
15. Jr FEH, Dupont with contributions from C, others many. Hmisc: Harrell Miscellaneous. 2020. https://CRAN.R-project.org/package=Hmisc.

16. Hunt RJ. Percent agreement, Pearson's correlation, and Kappa as measures of inter-examiner reliability. J Dent Res. 1986;65:128-30.

17. R Core Team. R: a language and environment for statistical computing. $R$ Foundation for Statistical Computing; 2019. https://www.R-project.org/.

18. Saleh R, Sasidharan Nair V, Toor SM, Taha RZ, Murshed K, Al-Dhaheri M, et al. Differential gene expression of tumor-infiltrating CD8+ T cells in advanced versus early-stage colorectal cancer and identification of a gene signature of poor prognosis. J Immunother Cancer. 2020;8:e001294.

19. Idos GE, Kwok J, Bonthala N, Kysh L, Gruber SB, Qu C. The prognostic implications of tumor infiltrating lymphocytes in colorectal cancer: a systematic review and meta-analysis. Sci Rep. 2020;10:3360.

20. Kumar S, Singh SK, Rana B, Rana A. Tumor-infiltrating CD8+ T cell antitumor efficacy and exhaustion: molecular insights. Drug Discov Today. 2021;26:951-67.

21. Sangaletti S, Chiodoni C, Tripodo C, Colombo MP. The good and bad of targeting cancer-associated extracellular matrix. Curr Opin Pharmacol. 2017;35:75-82.

22. Joyce JA, Fearon DT.T cell exclusion, immune privilege, and the tumor microenvironment. Science. 2015;348:74-80.

23. Lu P, Weaver VM, Werb Z. The extracellular matrix: a dynamic niche in cancer progression. J Cell Biol. 2012;196:395-406.

24. Failmezger H, Muralidhar S, Rullan A, de Andrea CE, Sahai E, Yuan Y. Topological tumor graphs: a graph-based spatial model to infer stromal recruitment for immunosuppression in melanoma histology. Cancer Res. 2020;80:1199-209.

25. Reichling C, Taieb J, Derangere V, Klopfenstein Q, Le Malicot K, Gornet $J-M$, et al. Artificial intelligence-guided tissue analysis combined with immune infiltrate assessment predicts stage III colon cancer outcomes in PETACC08 study. Gut. 2020;69:681-90.

26. van Pelt GW, Sandberg TP, Morreau H, Gelderblom H, van Krieken JHJM, Tollenaar RAEM, et al. The tumour-stroma ratio in colon cancer: the biological role and its prognostic impact. Histopathology. 2018;73:197-206.

27. Chu QD, Zhou M, Medeiros KL, Peddi P, Kavanaugh M, Wu X-C. Poor survival in stage IIB/C (T4N0) compared to stage IIIA (T1-2 N1, T1N2a) colon cancer persists even after adjusting for adequate lymph nodes retrieved and receipt of adjuvant chemotherapy. BMC Cancer. 2016;16:460.

28. Giraldo NA, Sanchez-Salas R, Peske JD, Vano Y, Becht E, Petitprez F, et al. The clinical role of the TME in solid cancer. Br J Cancer. 2019;120:45-53.

29. West NP, Dattani M, McShane P, Hutchins G, Grabsch J, Mueller W, et al. The proportion of tumour cells is an independent predictor for survival in colorectal cancer patients. Br J Cancer. 2010;102:1519-23.

30. van Pelt GW, Krol JA, Lips IM, Peters FP, van Klaveren D, Boonstra JJ, et al. The value of tumor-stroma ratio as predictor of pathologic response after neoadjuvant chemoradiotherapy in esophageal cancer. Clin Transl Radiat Oncol. 2020;20:39-44.

\section{Publisher's Note}

Springer Nature remains neutral with regard to jurisdictional claims in published maps and institutional affiliations.

Ready to submit your research? Choose BMC and benefit from:

- fast, convenient online submission

- thorough peer review by experienced researchers in your field

- rapid publication on acceptance

- support for research data, including large and complex data types

- gold Open Access which fosters wider collaboration and increased citations

- maximum visibility for your research: over 100M website views per year

At BMC, research is always in progress.

Learn more biomedcentral.com/submissions 